\title{
Scleral lens for keratoconus: technology update
}

\author{
Varsha M Rathi' \\ Preeji S Mandathara ${ }^{2}$ \\ Mukesh Taneja' \\ Srikanth Dumpati' \\ Virender S Sangwan' \\ 'L V Prasad Eye Institute, Hyderabad, \\ India; ${ }^{2} \mathrm{School}$ of Optometry and \\ Vision Science, University of New \\ South Wales, Kensington, NSW, \\ Australia
}

This article was published in the following Dove Press journal:

Clinical Ophthalmology

28 October 2015

Number of times this article has been viewed

Abstract: Scleral lenses are large diameter lenses which rest over the sclera, unlike the conventional contact lenses which rest on the cornea. These lenses are fitted to not touch the cornea and there is a space created between the cornea and the lens. These lenses are inserted in the eyes after filling with sterile isotonic fluid. Generally, scleral contact lenses are used for high irregular astigmatism as seen in various corneal ectatic diseases such as keratoconus, pellucid marginal degeneration, or/and as liquid bandage in ocular surface disorders. In this article, we review the new developments, that have taken place over the years, in the field of scleral contact lenses as regard to new designs, materials, manufacturing technologies, and fitting strategies particularly for keratoconus.

Keywords: keratoconus, scleral lens, technology update, PROSE

\section{Introduction}

Technological advances have occurred in many fields related to scleral contact lenses (ScCLs) which include contact lens materials, designs, and manufacturing techniques. ${ }^{1}$ $\mathrm{ScCL}$ is one of those fields which has actually seen resurrection after being dormant for almost about a century. Eugene Kalt, August Muller, and Eugen Fick individually made the first contact lenses of glass shells way back in $1880 \mathrm{~s}^{2-4}$ With the introduction of polymethyl methacrylate (PMMA) material in 1930, scleral lens came into general use. However, use of PMMA material led to corneal edema in contact lens users, and they experienced colored haloes causing Sattler's veil. Later on, small diameter corneal contact lenses became available in the late 1940s, but it was not before 1960 that the importance of oxygen permeability of contact lens material in preventing corneal edema was known. ${ }^{5}$ With the availability of corneal lenses, the interest in scleral lenses dwindled until Donald Ezekiel introduced oxygen-permeable ScCL which had greater comfort and acceptability among the users. ${ }^{6}$ Further developments in lens materials, designs, and use of new technology in making these lenses and also lens fitting techniques have led to better acceptability of scleral lenses. With the advancement in the various areas of technology, 21 st century has seen many new developments in contact lenses that has resulted in a greater interest and wider acceptance in the use of these lenses for patients' benefit.

These lenses are used for improving vision in patients with high or irregular astigmatism such as keratoconus, pellucid marginal degeneration, keratoglobus, and post-keratoplasty astigmatism. The other indication for these lenses is in patients having ocular surface disease such as Stevens-Johnson syndrome, dry eyes, graft-vs-host disease, and ocular cicatricial pemphigoid. ${ }^{7,8}$

Keratoconus is characterized by a presence of corneal ectasia and thinning that results in high irregular astigmatism and thereby poor vision. ${ }^{9}$ In mild to moderate cases, soft and rigid gas permeable (RGP) lenses may help to improve vision, but as the conicity of cornea increases, these lenses do not improve vision and patients may
Correspondence: Varsha M Rathi L V Prasad Eye Institute, L V Prasad Marg, Banjara Hills, Hyderabad 500034, Telangana State, India Email varsharathi@lvpei.org 
have to use other lenses such as piggyback contact lenses, hybrid lenses, and ScCL. ${ }^{10}$ Before we go into the details of the technology update, we need to know the basic design of scleral lenses.

ScCL are lenses that rest on sclera and do not touch the cornea with a space present between scleral lens and cornea. This is termed as corneal clearance of scleral lenses and is due to vaulting of lens (Figure 1). The lens is filled with fluid prior to insertion in the eye. The fluid can be unpreserved saline or normal saline ( $0.9 \%$ sodium chloride). ${ }^{11,12}$

These lenses consist of three parts, scleral (haptic) portion that rests on sclera, vault, that is responsible for corneal and limbal clearance of the lens; and optical portion of the lens. The optical portion of scleral lenses is usually $0.2 \mathrm{~mm}$ larger than the horizontal visible iris diameter. However, while fitting most of the scleral lenses, more attention is given to the haptic portion, the corneal (and limbal) clearance, and the edge of the lens. All these factors affect the "on-eye" performance of scleral lenses. When fitted, the lens should not move on to the eye. ${ }^{13}$

Various articles have shown improvement in visual acuity with ScCL. In keratoconus, the presence of higher-order aberrations reduces visual acuity; this can be improved further by adding front surface eccentricity (FSE) or toricity or wave front aberrations correction to spherical scleral lens. ${ }^{14}$ This review talks about the technology update in the field of ScCL that has improved the use of the lenses for patients with keratoconus with the aim of improving visual acuity and achieving a comfortable fitting without compromising the ocular surface. These advances are in lens materials, designs, manufacturing of these lenses, improved on-eye fitting techniques, and a better understanding of keratoconus per se.

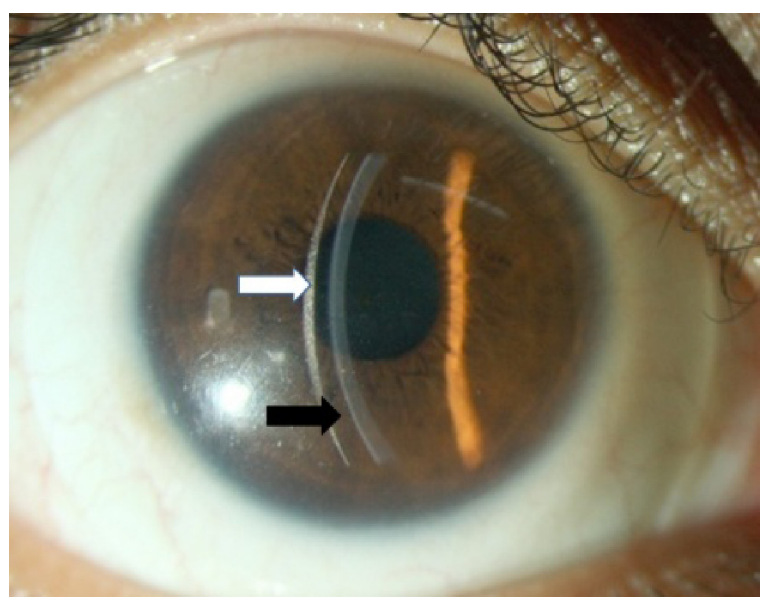

Figure I Corneal clearance on slit lamp biomicroscopy.

Note: Scleral lens (white arrow) with haptic resting on sclera and the space between the scleral lens and the cornea - corneal clearance (black arrow).

\section{Scleral lens designs}

The scleral lenses can be air-ventilated (fenestrated) or fluidventilated (non-fenestrated). ${ }^{15}$ This ventilation helps in providing oxygen to the ocular surface without compromising the physiology. With fenestrated lenses, there is a possibility for the air bubble coming into the visual axis and compromising visual acuity, but this is not so with the fluid-ventilated lenses. Rosenthal and Croteau had shown the use of fluid-ventilated scleral lenses in patients of keratoconus. ${ }^{4}$ The ventilation can be done with channels that are added to the scleral lenses. These are nonpenetrating grooves which make extra tears available, but results in less tear exchange. Improved oxygen availability is possible with the high Dk material. Dk defines the oxygen permeability of the lens material. It is the inherent property of material; $\mathrm{D}$ is diffusion coefficient and $\mathrm{k}$ represents the oxygen solubility of a contact lens material. Lens thickness determines the oxygen transmissibility.

Scleral lenses are lenses with bearing only on the sclera with diameter of the lens being $15 \mathrm{~mm}$ and above. ${ }^{16}$ Miniscleral have diameter between 15 and $18 \mathrm{~mm}$ and true scleral lenses have $>18 \mathrm{~mm}$ diameter (more than $6 \mathrm{~mm}$ bearing on sclera). Mini-scleral have less corneal clearance as compared to true sclerals. Various lenses available include mini-scleral design (MSD), Maxim, Jupiter, Boston mini-scleral, and Tru-scleral. The true scleral lenses are Jupiter, Pullum lenses, Tru-scleral, and PROSE (prosthetic replacement of the ocular surface ecosystem) (Boston Foundation for Sight, Needham Heights, MA, USA). Small diameter lenses are prone for getting stuck to the cornea due to suction vacuum and may pose a challenge to the fitter. ${ }^{17}$ This suction is reduced or eliminated by air through fenestrations or fluid ventilation by making grooves/channels on posterior surface. Patients do sometimes find it difficult to handle mini-scleral lenses for lens insertion and removal. Table 1 shows some of these lenses.

True scleral lens - various companies provide these lenses. PROSE is one such lens provided by Boston Foundation for Sight.

\section{Toricity and scleral lenses}

Scleral lenses mask the irregular astigmatism with the fluid reservoir. Most important aspect in fitting scleral lenses is aligning the haptic to the sclera. Some patients do have edge lifts indicating toric nature of sclera and this toricity can be added to scleral lens. This is possible with the availability of submicron lathe machines or computerized lathe machines. This reduces the amount of debris collection between the lens and the cornea. In addition, the comfort of the patients increases along with improved wear time. ${ }^{18}$ 
Table I Description of mini-scleral lenses available in the market

\begin{tabular}{|c|c|c|}
\hline Name (manufacturer) & Diameter & Description \\
\hline $\begin{array}{l}\text { MSD (Blanchard Contact Lens Inc, } \\
\text { Manchester, UK) }\end{array}$ & $15.8 \mathrm{~mm}$ & $\begin{array}{l}\text { Aspheric front surface and reverse geometry posterior surface } \\
\text { with specially designed posterior and optical curves. Aspheric } \\
\text { front surface reduces aberrations and improves vision }\end{array}$ \\
\hline Jupiter (Visionary Optics, Front Royal, VA, USA) & $15.0-18.2 \mathrm{~mm}$ & Manufactured with focal point technology \\
\hline \multicolumn{3}{|l|}{ Medlens Innovations, Front Royal, VA, USA/ } \\
\hline \multicolumn{3}{|l|}{ Essilor contact lens, Dallas TX, USA) } \\
\hline $\begin{array}{l}\text { Europa lens (Next generation of Jupiter scleral } \\
\text { lens from Visionary Optics) }\end{array}$ & $16.0-18.0 \mathrm{~mm}$ & Reverse geometry design with increased optic zone \\
\hline $\begin{array}{l}\text { Boston mini-scleral (Boston Foundation } \\
\text { for Sight, Needham Heights, MA, USA) }\end{array}$ & $\begin{array}{l}15 \mathrm{~mm} \text { and } \\
\text { more }\end{array}$ & Custom-designed lenses using computerized lathe machine \\
\hline \multicolumn{2}{|l|}{ San Antonio, TX, USA) } & Have radial channels into periphery \\
\hline Innovative sclerals & $15.0-233.0 \mathrm{~mm}$ & Fitted on sag height of cornea \\
\hline
\end{tabular}

Abbreviation: MSD, mini-scleral design.

\section{Materials}

Current scleral lenses are manufactured with high oxygen permeable materials such as fluorosilicone acrylate as compared to old PMMA materials though some papers have described the use of PMMA materials. ${ }^{15}$ With the availability of high Dk material, the oxygen supply to cornea is improved. Also, the scleral lens with a greater vault may have more fluid in it. Pullum and Stapleton had shown that there is less than $3 \%$ of corneal edema in normal eyes after 3 hours of lens wear for a scleral lens of $0.6 \mathrm{~mm}$ thickness and a Dk value of $115 \times 10^{-11} \mathrm{~cm}^{2} \mathrm{~mL} \mathrm{O}_{2 / \mathrm{s}} \mathrm{mL} \mathrm{mm} \mathrm{Hg}$ (ISO/FAT). ${ }^{19}$ Smith et al had shown the development of corneal edema when the lens thickness was $0.8 \mathrm{~mm}$ and a Dk value of $80 .{ }^{20}$ However, a further increase in Dk value was not associated with improved outcome. ${ }^{19}$ There are various materials available such as Boston EO (Dk 82), XO (Dk 100), and Menicon Z which have high Dk values, good dimensional stability, minimal lens flexure, and greater oxygen transmissibility. It is possible to make thin lens designs with the newer software which are now available for making scleral lenses. With high Dk material, wettability of contact lenses may be reduced which may reduce patients comfort. Plasma coating of the surface of ScCL improves surface wettability and thereby comfort and allows improved daily lens wear. ScCL do not cause corneal edema. However, ScCL need to be in highest Dk material and tear lens should not be excessive to prevent hypoxia. ${ }^{21}$

Bergmanson et al in his editorial, however, stated that corneal hypoxia occurring from wearing modern scleral lenses has neither been proven nor disproven. ${ }^{22}$

\section{Manufacturing of scleral lenses}

Scleral lenses were earlier manufactured through the molding process (impression method). Impression technique is not used nowadays in modern scleral lens practice as it is cumbersome. ${ }^{8}$ In impression technique, a mold (cast) is made of the anterior ocular surface and then the cast is sent to the manufacturer for making the lens. ${ }^{8,15}$

With preformed (trial fitting) lenses, trial sets are available which helps practitioner fit the ScCL. Nowadays, this is the most preferred method of scleral lens fitting. Various companies manufacture these lenses in various designs and give a fitting guide for the practitioners. With the advent of computerized lathe cut machines, it is possible to make lenses with submicron precision. PROSE is a scleral lens which uses a computer-assisted design and manufacture (CAD/ CAM) software for lens manufacturing and makes lenses with precision $^{1}$ (Figure 1). Today scleral lenses are made to have a very smooth surface, smoother edges, and very low errors in making. Contact lens practitioners now can design or make changes in the design themselves on a computer after assessing the fit during trials. PROSE lenses are manufactured using Optoform lathe system for spherical lenses and Nanoform lathe machines for lenses with toricity at Boston Foundation for Sight. The computer design can be sent to the base unit for manufacturing the lenses with the help of software, allowing for a faster delivery to the end users.

Until now, corneal clearance is assessed on slit lamp examination either by comparing it with the known thickness of the lens that is used as trial or by comparing it with the corneal thickness of the patient which at best gives only a rough estimation. With the availability of newer technology, such as anterior segment optical coherence tomography (ASOCT), it is possible to measure the amount of corneal clearance much more accurately (Figure 2). Gemoules has described the role of OCT in defining the relationship of contact lenses and the eye. ${ }^{23}$ Our experience with OCT showed that the mean value 


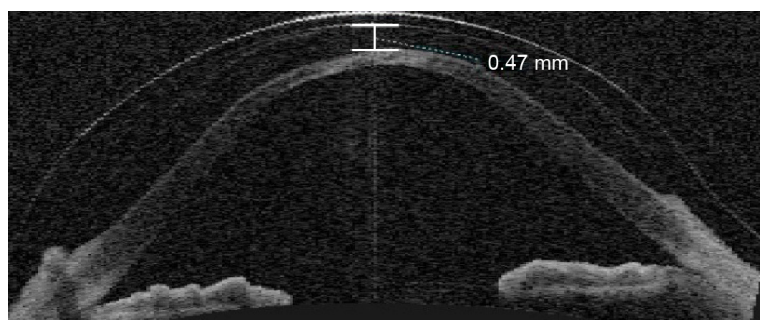

Figure 2 ASOCT in a patient with keratoconus with vault measured as $0.47 \mathrm{~mm}$. Note: The vault is more in periphery as compared to the center. Abbreviation: ASOCT, anterior segment optical coherence tomography.

of height of vault after 1-hour challenge with scleral lenses was $0.80 \mathrm{~mm}$ and reduced to $0.54 \mathrm{~mm}$ after 4-hour challenge. Before finalizing the fitting, this reduction in corneal clearance needs to be taken into consideration. This is especially important in progressive disease such as keratoconus, lenses with higher vaults can be worn for longer days even if the cone progresses. If the vault is less, with the progression of cone, the lens may cause corneal touch resulting in poor vision and scarring. Sonsino and Mathe have shown that there is no correlation between change in vault or fluid and visual acuity in patients with dry eyes. ${ }^{24}$

By increasing the sagittal height and thereby vault, one can use these lenses over a prolonged period without compromising vision. Kauffman et al have shown different settling rates of vault with three different scleral lens designs in a short-term study. ${ }^{25}$

\section{Scleral or haptic portion}

Fitting of scleral lens needs a different approach as compared to corneal RGP fitting strategy wherein corneal topography can be used as an aid for fitting lenses. However, corneal topography does not aid in fitting scleral lenses. ${ }^{26}$ Scleral lens rests on sclera and proper alignment of scleral portion of lens to sclera is of prime importance. Measurement of scleral shape is not possible though OCT can aid in knowing the corneo-scleral shape. OCT can measure up to $16 \mathrm{~mm}$ of cornea and if the machine is decentered, one can measure up to $20 \mathrm{~mm}$ of the anterior ocular surface..$^{27,28}$ PROSE lenses incorporates the spline technology which helps in designing the haptic to be tailor-made for each patient. ${ }^{29}$ While fitting traditional RGP contact lenses on an eye, the lens has more central curvature and flatter periphery. This junction usually appears nodular without any blending. The spline technology creates a junction-less surface, which makes it possible to fit these lenses on any ocular surface without causing any damage, impingement, or compression on the ocular surface. ${ }^{29}$ The fitting of smaller diameter scleral lenses poses challenges as the smaller haptic rests on smaller area causing focal scleral compression and may result in the suction of the lenses on the eye making the patient uncomfortable. ${ }^{30}$ However, for keratoconus, large diameter lenses are preferred for even weight bearing and reducing suction on the eye.

\section{Front surface eccentricity}

PROSE lenses are available in different FSE values of 0.3 , 0.6 , and 0.8. A spherical lens has FSE of 0. The higher FSE values indicate rapid flattening of the lens from the center toward the periphery. ${ }^{31}$ PROSE with 0.6 and 0.8 FSE can improve vision in patients with advanced keratoconus. ${ }^{14}$ Hussoin et al have proposed various mechanisms for the role of FSE. FSE may compensate for aberrations from the posterior surface, this may compensate for poor alignment of the optical axis of patient and of the lens, and this eccentricity may restore prolate surface thus improving vision. ${ }^{14}$

\section{Vault}

Vault of scleral lenses is not associated with a change in visual acuity or fitting of the lens and can be changed irrespective of rest of the scleral portion of lenses. This is especially so in PROSE lenses.

\section{Fenestrations}

The earlier scleral lenses had fenestrations for providing oxygen delivery to the eye and for tear exchange. With fenestrations, the vault can be decreased from 150 to $40 \mu$. With the availability of high Dk material, fenestrations are not required in modern-day scleral lens practice as oxygen transmissibility is improved. These are not preferred nowadays and are used on a case-by-case basis and these can be used for postkeratoplasty contact lens fitting for high astigmatism or ectasia in the graft.

\section{Scleral lenses do not move on the eye}

Ticak et al have shown with three scleral lens designs that were designed at the Visual Optics Institute that the lens remains stable on the eye and do not move and can be useful for correction of aberrations. ${ }^{32}$ This helps in designing the lenses for customized wavefront correction of highly aberrated eyes of patients having keratoconus. ${ }^{31,32}$ Sabesan et al and Marsack et al have shown that wavefront-guided (WFG) optics can be incorporated in scleral lenses to correct higher-order aberrations and thereby improve vision. ${ }^{33,34}$ Shi et al had shown that it is possible to improve the average visual acuity by optimizing WFG correction in the presence of registration uncertainty in patients with keratoconus. ${ }^{35}$ 
Sabesan et al have fitted spherical lenses first and then corrected the aberrations of the eye on the lens surface with a submicron precision lathe machine and used the lens for better "on-eye" performance. ${ }^{33}$ The high contrast visual acuity with these WFG ScCL lenses did not reach the agematched visual acuity. ${ }^{34}$ Marsack et al have shown that if the lenses move on eye, the optical quality and vision is reduced. ${ }^{34}$ Sabesan et al had shown that though the initial improvement in visual acuity is less with WFG lenses, visual acuity improves over time as the patients adapt to the improved retinal image. ${ }^{33}$

\section{PROSE}

PROSE, which was earlier called as Boston scleral lens or Boston scleral lens prosthetic device or Boston ocular surface prosthesis, is a computer-assisted custom-designed lens that is made using a computerized lathe machine. ${ }^{4}$ It uses spline technology which created junction-less surface of the haptic that aligns with sclera and minimizes scleral indentation, compression, or impingement. Channels can be added to the lens as desired. The spline function allows the creation of lens with great precision and reproducibility. ${ }^{4}$ These lenses have been shown to be effective in various studies done on patients with corneal ectasia including keratoconus for improving both the vision and comfort. ${ }^{11,17,36}$ These lenses have an added feature of FSE which improves vision even in advanced keratoconus. ${ }^{14}$

Scleral lenses in keratoconus are rarely associated with infectious keratitis or other adverse events..$^{37,38}$ We assume that patients with ocular surface disease may be more prone to these adverse events compared to keratoconus. This can be due to poor cleaning of the plunger used for the insertion and removal of these lenses, improper use of saline solution, or patient's poor hygiene. Proper care regimen including mechanical cleaning is required to clean these lenses though one-step hydrogen peroxide is used as a disinfecting system.

With improvement in technological advances with presbyopic lenses, today scleral lenses are also available for elderly keratoconus patients with presbyopic correction. Maxim scleral lens is a scleral lens for advanced keratoconus with near add design. However, literature on use of these lenses is sparse. Fitting of scleral lenses is possible after intracorneal ring segments implantation in patients having keratoconus (Figure 3).

There remains few hurdles in manufacturing these customdesigned lenses as this demands specialized equipment. The cost of the precision lathe machines is very high, which

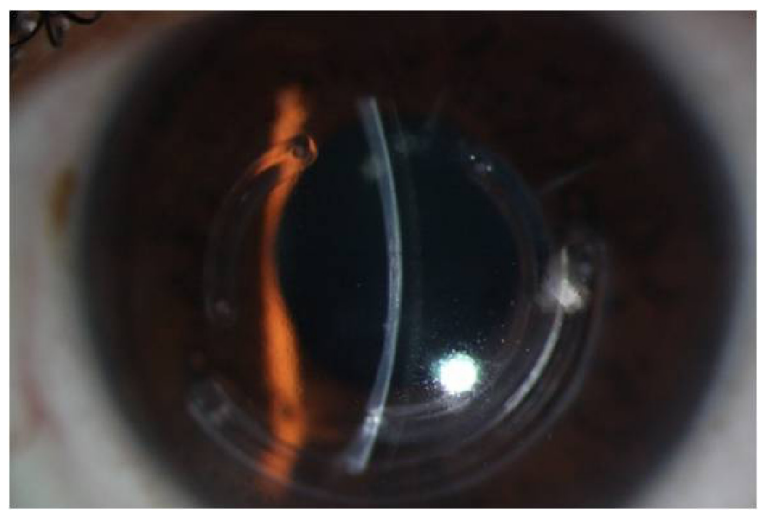

Figure 3 PROSE in keratoconus.

Notes: Scleral lens in keratoconus patient having intracorneal rings. The patient was referred for keratoplasty as he was not able to wear RGP lenses. With PROSE having FSE of 0.6, his visual acuity improved to 20/20.

Abbreviations: FSE, front surface eccentricity; PROSE, prosthetic replacement of the ocular surface ecosystem; RGP, rigid gas permeable.

increases the cost of manufacturing these lenses significantly. High cost and the special training required in assessing the fitting and then modifying the design is quite challenging. Still, with the advances in technology, the field of ScCL has led to its resurgence as specialized lenses. Despite these challenges, improvement in visual acuity with scleral lenses can bring down the rate of keratoplasty in patients of keratoconus and thereby significantly reduce the cost, effort, and other issues related to the maintenance of corneal grafts. ${ }^{39}$

\section{Disclosure}

The authors report no conflicts of interest in this work.

\section{References}

1. Rosenthal P. The Boston Lens and the management of keratoconus. Int Ophthalmol Clin. 1986;26(1):101-109.

2. Romero-Rangel T, Stavrou P, Cotter J, Rosenthal P, Baltatzis S, Foster CS. Gas-permeable scleral contact lens therapy in ocular surface disease. Am J Ophthalmol. 2000;130(1):25-32.

3. Rosenthal P, Cotter J. The Boston Scleral Lens in the management of severe ocular surface disease. Ophthalmol Clin North Am. 2003;16(1): 89-93.

4. Rosenthal P, Croteau A. Fluid-ventilated, gas-permeable scleral contact lens is an effective option for managing severe ocular surface disease and many corneal disorders that would otherwise require penetrating keratoplasty. Eye Contact Lens. 2005;31(3):130-134.

5. The IACLE Contact lens course: Module 9: 145-146 (First edition).

6. Ezekiel D. Gase permeable haptic lenses. J Br Contact Lens Assoc. 1983;6: 158-161.

7. Schornack MM. Scleral lenses: a literature review. Eye Contact Lens. 2015;41(1):3-11.

8. vanderWorp E, Bornman D, Ferreira DL, Faria-Ribeiro M, Garcia-Porta N, Gonzalez-Meijome JM. Modern scleral contact lenses: a review. Cont Lens Anterior Eye. 2014;37(4):240-250.

9. Krachmer JH, Feder RS, Belin MW. Keratoconus and related noninflammatory corneal thinning disorders. Surv Ophthalmol. 1984;28(4): 293-322.

10. Rathi VM, Mandathara PS, Dumpati S. Contact lens in keratoconus. Indian J Ophthalmol. 2013;61(8):410-415. 
11. Rathi VM, Mandathara PS, Dumpati S, Vaddavalli PK, Sangwan VS. Boston ocular surface prosthesis: an Indian experience. Indian J Ophthalmol. 2011;59(4):279-281.

12. Jacobs DS. Update on scleral lenses. Curr Opin Ophthalmol. 2008; 19(4):298-301.

13. Jacobs DS, Rosenthal P. Boston scleral lens prosthetic device for treatment of severe dry eye in chronic graft-versus-host disease. Cornea. 2007;26(10):1195-1199.

14. Hussoin T, Le HG, Carrasquillo KG, Johns L, Rosenthal P, Jacobs DS. The effect of optic asphericity on visual rehabilitation of corneal ectasia with a prosthetic device. Eye Contact Lens. 2012;38(5):300-305.

15. Pullum KW, Whiting MA, Buckley RJ. Scleral contact lenses: the expanding role. Cornea. 2005;24(3):269-277.

16. Barnett M, Mannis MJ. Contact lenses in the management of keratoconus. Cornea. 2011;30(12):1510-1516.

17. Rathi VM, Mandathara PS, Vaddavalli PK, Srikanth D, Sangwan VS Fluid filled scleral contact lens in pediatric patients: challenges and outcome. Cont Lens Anterior Eye. 2012;35(4):189-192.

18. Visser ES, Visser R, Van Lier HJ. Advantages of toric scleral lenses. Optom Vis Sci. 2006;83(4):233-236.

19. Pullum KW, Stapleton FJ. Scleral lens induced corneal swelling: what is the effect of varying Dk and lens thickness? CLAO J. 1997;23(4): 259-263.

20. Smith GT, Mireskandari K, Pullum KW. Corneal swelling with overnight wear of scleral contact lenses. Cornea. 2004;23(1):29-34.

21. Jaynes JM, Edrington TB, Weissman BA. Predicting scleral GP lens entrapped tear layer oxygen tensions. Cont Lens Anterior Eye. 2015; 38(1):44-47.

22. Bergmanson JP, Ezekiel DF, van der Worp E. Scleral contact lenses and hypoxia: theory versus practice. Cont Lens Anterior Eye. 2015;38(3): 145-147.

23. Gemoules G. A novel method of fitting scleral lenses using high resolution optical coherence tomography. Eye Contact Lens. 2008;34(2): 80-83.

24. Sonsino J, Mathe DS. Central vault in dry eye patients successfully wearing scleral lens. Optom Vis Sci. 2013;90(9):e248-e251; discussion 1030 .

25. Kauffman MJ, Gilmartin CA, Bennett ES, Bassi CJ. A comparison of the short-term settling of three scleral lens designs. Optom Vis Sci. 2014; 91(12):1462-1466.
26. Schornack MM, Patel SV. Relationship between corneal topographic indices and scleral lens base curve. Eye Contact Lens. 2010;36(6): 330-333.

27. van der Worp E, Graf T, Caroline P. Exploring beyond the borders. Contact Lens Spectrum. 2010;6:26-32.

28. Kojima R, Caroline P, Graff T, et al. Eye shape and scleral lenses. Contact Lens Spectrum. 2013;3:8-43.

29. Rosenthal P, Cotter JM. Clinical performance of a spline-based apical vaulting keratoconus corneal contact lens design. CLAO J. 1995; 21(1):42-46.

30. Rosenthal P. Scleral lens development: evolution of an ocular surface prosthesis. Contact Lens Spectr. 2009;24(12):32-38.

31. Gumus K, Gire A, Pflugfelder SC. The impact of the Boston ocular surface prosthesis on wavefront higher-order aberrations. Am J Ophthalmol. 2011;151(4):682-690.e2.

32. Ticak A, Marsack JD, Koenig DE, et al. A comparison of three methods to increase scleral contact lens on-eye stability. Eye Contact Lens. Epub 2015 May 2.

33. Sabesan R, Johns L, Tomashevskaya O, Jacobs DS, Rosenthal P, Yoon G. Wavefront-guided scleral lens prosthetic device for keratoconus. Optom Vis Sci. 2013;90(4):314-323.

34. Marsack JD, Ravikumar A, Nguyen C, et al. Wavefront-guided scleral lens correction in keratoconus. Optometry Vision Sci. 2014;91(10): 1221-1230.

35. Shi Y, Queener HM, Marsack JD, Ravikumar A, Bedell HE, Applegate RA. Optimizing wavefront-guided corrections for highly aberrated eyes in the presence of registration uncertainty. J Vis. 2013;13(7).

36. Rathi VM, Sudharman Mandathara P, Vaddavalli PK, Dumpati S, Chakrabarti T, Sangwan VS. Fluid-filled scleral contact lenses in vernal keratoconjunctivitis. Eye Contact Lens. 2012;38(3):203-206.

37. Bruce AS, Nguyen LM. Acute red eye (non-ulcerative keratitis) associated with mini-scleral contact lens wear for keratoconus. Clin Exp Optom. 2013;96(2):245-248.

38. Zimmerman AB, Marks A. Microbial keratitis secondary to unintended poor compliance with scleral gas-permeable contact lenses. Eye Contact Lens. 2014;40(1):e1-e4.

39. DeLoss KS, Fatteh NH, Hood CT. Prosthetic Replacement of the Ocular Surface Ecosystem (PROSE) scleral device compared to keratoplasty for the treatment of corneal ectasia. Am J Ophthalmol. 2014;158(5): 974-982.
Clinical Ophthalmology

\section{Publish your work in this journal}

Clinical Ophthalmology is an international, peer-reviewed journal covering all subspecialties within ophthalmology. Key topics include: Optometry; Visual science; Pharmacology and drug therapy in eye diseases; Basic Sciences; Primary and Secondary eye care; Patient Safety and Quality of Care Improvements. This journal is indexed on Submit your manuscript here: http://www.dovepress.com/clinical-ophthalmology-journal
Dovepress

PubMed Central and CAS, and is the official journal of The Society of Clinical Ophthalmology (SCO). The manuscript management system is completely online and includes a very quick and fair peer-review system, which is all easy to use. Visit http://www.dovepress.com/ testimonials.php to read real quotes from published authors. 